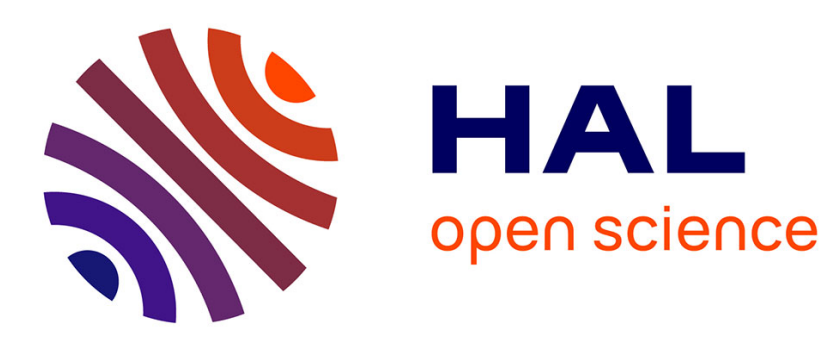

\title{
Radial thermal conductivity of a PAN type carbon fiber using the 3 omega method
}

\author{
Rodrigo Andres Olaya Gomez, Bertrand Garnier
}

\section{To cite this version:}

Rodrigo Andres Olaya Gomez, Bertrand Garnier. Radial thermal conductivity of a PAN type carbon fiber using the 3 omega method. International Journal of Thermal Sciences, 2022, 172, pp.107321. 10.1016/j.ijthermalsci.2021.107321 . hal-03430963

\section{HAL Id: hal-03430963 https://hal.science/hal-03430963}

Submitted on 16 Nov 2021

HAL is a multi-disciplinary open access archive for the deposit and dissemination of scientific research documents, whether they are published or not. The documents may come from teaching and research institutions in France or abroad, or from public or private research centers.
L'archive ouverte pluridisciplinaire HAL, est destinée au dépôt et à la diffusion de documents scientifiques de niveau recherche, publiés ou non, émanant des établissements d'enseignement et de recherche français ou étrangers, des laboratoires publics ou privés. 


\title{
Radial thermal conductivity of a PAN type carbon fiber using the 3omega method
}

\author{
Olaya Rodrigo, Bertrand Garnier \\ CNRS, LTeN UMR 6607, Université de Nantes, Rue Christian Pauc, 44306 Nantes Cedex 3, France \\ Corresponding author: Rodrigoandres.Olayagomez@univ-nantes.fr
}

\begin{abstract}
The prediction of the effective thermal conductivity of composites filled with carbon fibers requires the knowledge of the microstructure and composition of the composite, the orientation of nonisometric filler, the thermal conductivities of both phases and the thermal contact resistances between fibers and also between fibers and matrix. Due to the anisotropy of carbon fibers, one should know both their axial and radial thermal conductivities. Contrary to the axial thermal conductivity of carbon fiber, there are not much work on the radial one. The present work describes the characterization of the thermal conductivity of carbon fiber in the radial direction using the 3 omega method with a constant current source. One key point is the use of de-ionized water around the carbon fibers to enhance radial heat transfer. An appropriate thermal model is required in order to estimate the radial thermal conductivity. Therefore, analytical 1D and 2D thermal models are developed using quadrupole methods to describe heat transfer in the carbon fiber using periodic regime and are compared with a 2D numerical model. It appeared that the use of a 1D heat transfer model induces some bias until $50.3 \%$ on the estimation of the radial thermal conductivity showing that residual axial heat transfer still occurs. Therefore the $2 \mathrm{D}$ thermal model is more appropriate and is used with the experimental data to estimate the radial thermal conductivity. In addition, a detailed sensitivity analysis of the unknown parameter is performed that allows to find the best range of operating conditions especially the frequency range and the effect of the type of surrounding material. Measurements are performed with PAN type carbon fiber (FT300B) of 6 to 8 micrometers diameter and various lengths from 0.5 to $2.5 \mathrm{~mm}$ embedded in de-ionized water. Finally, radial thermal conductivity values are shown to be about 10 times smaller than the axial one, revealing strong anisotropy of the studied carbon fiber.
\end{abstract}

Keywords: radial thermal conductivity; carbon fiber; 3omega method; quadrupole method

\section{Nomenclature:}

C Heat capacity, $\mathrm{Jkg}^{-1} \mathrm{~K}^{-1}$

$I_{r m s} \quad$ Intensity of the current, A

$f \quad$ Frequency $\left(f=2 \pi \omega^{-1}\right), \mathrm{Hz}$

$k_{r} \quad$ Fiber radial therm. conduct., $\mathrm{Wm}^{-1} \mathrm{~K}^{-1}$

$k_{w} \quad$ Water thermal conductivity, $\mathrm{Wm}^{-1} \mathrm{~K}^{-1}$

$k_{Z} \quad$ Fiber axial therm. conduct., $\mathrm{Wm}^{-1} \mathrm{~K}^{-1}$

$L \quad$ Fiber length, $\mathrm{m}$

$R \quad$ Radius of the fiber, $\mathrm{m}$

$R_{0} \quad$ Elec. resistance of the fiber, $\Omega$

$T \quad$ Temperature, ${ }^{\circ} \mathrm{C}$

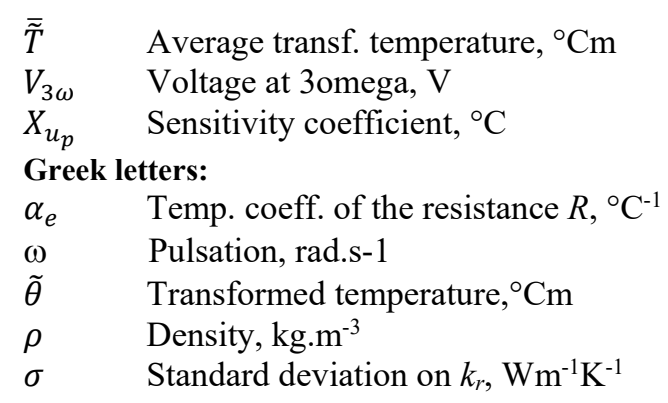

\section{Introduction}

Carbon fibers has reached a great place in many high-performance industries such as aerospace or automotive structures [1]. This is because carbon fiber has very specific characteristics such as high strength, light weight which makes polymeric material reinforced with carbon fiber good candidate to replace metallic parts for structural components. As during their use composite material are subject to thermal gradient, it is of interest to predict their effective thermal conductivity. For this purpose, the knowledge of thermal property of carbon fiber are required. A lot of research focused on the measurement of the axial thermal conductivity of single carbon fiber mainly because it is the primary thermal conductivity to be measured. However as mentioned by Huang [2], carbon fibers are rather anisotropic material depending on the type of precursors and on the thermal processing method. Therefore the knowledge of radial thermal conductivity is quite often required. The problem of the measurement of the radial thermal conductivity of a carbon fiber is very difficult because of its small diameter typically less than $10 \mu \mathrm{m}$. 
In literature dealing with thermal characterization of carbon fiber, several researches were focused on the measurement of the axial thermal conductivity and very few on the radial one. Table 1 shows axial and radial thermal conductivity values for several type of carbon fibers. For the radial thermal conductivity, one have found works only from Huang [2], Wang [3] and Liang [5] showing radial values mainly smaller than the axial ones. In these works, various techniques are used. Huang [2] has used molecular dynamic simulation to compute the radial conductivity $k_{r}$ of pitch based carbon fiber but no checking with measurement was proposed. Wang [3] has used Raman method to measure $k_{r}$ but a very large variation of measured values (from 0.11 to $8 \mathrm{Wm}^{-1} \mathrm{~K}^{-1}$ ) was obtained according to the location of the laser beam, this was explained by variation in the microstructure of the carbon fiber but it could also be due to the too low sensitivity of the temperature measurement to $k_{r}$. Liang [5] has used the 3 omega method and a 1D radial thermal model to obtain $k_{r}$ but as we will see later a 2D model is more appropriate. Therefore it appears that the correct measurement of radial thermal conductivity of carbon fiber is still challenging.

Table 1. Radial $\left(k_{r}\right)$, axial $\left(k_{z}\right)$ thermal conductivities and volumetric heat capacity $(\rho C)$ of various types of carbon fibers from the literature $(\mathrm{RT}=$ room temperature)

\begin{tabular}{llllllll}
\hline Carbon & Commercial & $k_{r}$ & $k_{z}$ & $\rho C$ & Temperature & Method & Ref. \\
Fiber & Ref. & $\mathrm{Wm}^{-1} \mathrm{~K}^{-1}$ & $\mathrm{Wm}^{-1} \mathrm{~K}^{-1}$ & $\mathrm{Jm}^{-3} \mathrm{~K}^{-1}$ & $\mathrm{~K}$ & & \\
\hline Lignin & - & $0.11-8$ & $1.4-2.15$ & - & $77-\mathrm{RT}$ & FET-Raman + TET tech. & {$[3]$} \\
Pitch & YSH-60A & 12 & - & - & $\mathrm{RT}$ & MD simulation & {$[2]$} \\
Pitch & - & - & 490 & - & $\mathrm{RT}$ & T-type & {$[4]$} \\
PAN & T650 & 1.5 & 13.7 & $1.9710^{6}$ & $\mathrm{RT}$ & $3 \omega$ & {$[5]$} \\
PAN & FT300B & - & 10.47 & $1.3710^{6}$ & RT & $3 \omega$ & {$[6]$} \\
PAN & FT300B & & 8.5 & & RT & Hot guarded plate & {$[7]$} \\
Rayon & TC2 & - & $5-12.5$ & - & $850-1800$ & Periodic heating & {$[8]$} \\
\hline
\end{tabular}

In our work one have used the $3 \omega$ method. The working principle of this technique is the following. An additional metallic layer or the specimen itself serves as a heater and at the same time as a temperature sensor. As its electrical resistance varies linearly with temperature, its average temperature over its length and thickness (or diameter) can be measured through the measurement of voltage between its two extremities. The Joule heating is modulated thanks to an alternating current with a pulsation $\omega$. The current will create a heating with a volumic power and therefore temperature fluctuation at $2 \omega$, this further leads to voltage fluctuation across the metallic layer or specimen with both $\omega$ and $3 \omega$ pulsations. Only the voltage at $3 \omega$ noted $V_{3 \omega}$ contains the information of the average temperature of the sensor. It is therefore important to reduce the contribution of the voltage at $1 \omega$ by a specific device in the setup such as a Wheatstone bridge or differential amplifiers. Then the voltage $V_{3 \omega}$ is used with an appropriate thermal model to estimate the unknown such as the thermal conductivity of the sample. This $3 \omega$ method was developed for thin film characterization (Cahill [9]) and was successfully used to measure thermal conductivity of metallic micro or nanowires (Lu [10], Xing [11], Ding [12]) or carbon fibers (Liu [13], Mishra [6]). It is also possible to estimate simultaneously the axial thermal conductivity and the volumic heat capacity of wires or carbon fibers since these two parameters does not show correlation within the frequency range used during the $3 \omega$ measurement [6], typically from 1 to $1000 \mathrm{~Hz}$.

The objective of this work is to develop a method to provide radial thermal conductivity of carbon fiber. First the proposed measurement principle is presented then analytical thermal models are developed in order to compute $V_{3 \omega}$ values useful for the $k_{r}$ estimation procedure. First a 1D thermal model and then a 2D one are developed using analytical methods and both are compared with a 2D numerical model (finite elements). The sensitivity analysis versus frequency shows the interest of using de-ionized water for radial thermal conductivity measurement. Finally, $k_{r}$ measurements are performed with three different carbon fibers of PAN type.

\section{Radial thermal conductivity measurement principle}

Figure 1 shows the sample holder with the carbon fiber to be characterized radially. The carbon fiber of a few millimeter length (typically between 0.5 and $2 \mathrm{~mm}$ ) is connected to two copper blocks using silver paste as for previous axial thermal conductivity measurement (Mishra [6]). However, instead of placing the sample holder under vacuum (for axial characterization), it is immersed in deionized water, the aim being the increase of the radial thermal gradient inside the carbon fiber and also the decrease of the axial one. Then as for classical 3omega 
measurement a modulated current is applied between the two extremities of the sample that are connected to two differential amplifiers then to a lock-in amplifier. The interest of the use of deionized water will be detailed in the section 3.3 dedicated to sensitivity analysis. Next section will describe the thermal models needed to estimate $k_{r}$ from the knowledge of the amplitude of the average temperature of the carbon fiber and we will show the relationship between the latter and the measured voltage $V_{3 \omega}$.

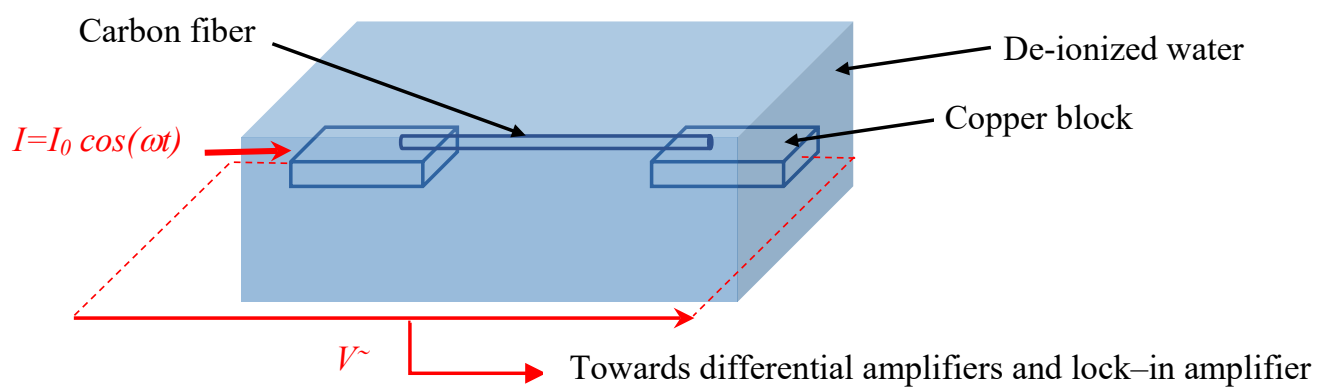

Fig. 1. Carbon fiber radial thermal conductivity measurement principle using $3 \omega$ method

\section{Thermal models}

\subsection{D analytical model}

This section provides an analytical 1D radial thermal model for the self-heating of carbon fiber surrounded by deionized water. Fig. 2 shows the geometry that has been considered. A volumic power $P$ due to Joule effect is located within the carbon fiber and the surrounding material (water) is considered as a semi -infinite medium. In our work $P$ can be assumed constant since the current is prescribed and the electrical resistance of the carbon fiber has a very small temperature coefficient. For the other cases, Ding [12] has proposed a criterion to check if the hypothesis $P$ constant can be considered.

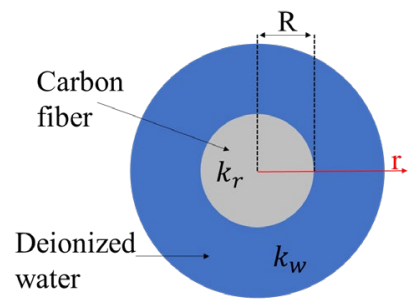

Fig. 2. Geometry for the 1D thermal model with a carbon fiber surrounded by de-ionized water

Therefore, the corresponding radial heat equations for carbon fiber and deionized water can be expressed as follow:

$$
\begin{gathered}
k_{r}\left(\frac{\partial^{2} T}{\partial r^{2}}+\frac{1}{r} \frac{\partial T}{d r}\right)-\rho c \frac{\partial T}{\partial t}=-P \\
k_{w}\left(\frac{\partial^{2} T_{w}}{\partial r^{2}}+\frac{1}{r} \frac{\partial T_{w}}{d r}\right)-\rho_{w} c_{w} \frac{\partial T_{w}}{\partial t}=0
\end{gathered}
$$

where $T$ and $T_{w}$ are the temperature inside respectively the carbon fiber and the water, $\rho c$ is the volumetric heat capacity of carbon fiber, $k_{w}$ and $\rho_{w} c_{w}$ are the thermal conductivity and the volumetric heat capacity of water.

The internal and external boundary conditions and initial conditions are: 


$$
\left\{\begin{array}{c}
\text { at } r=R: T=T_{w} \text { and }\left.k_{r} \frac{\partial T}{\partial r}\right|_{R}=\left.k_{w} \frac{\partial T_{w}}{\partial r}\right|_{R} \text { (perfect contact) } \\
\text { at } r=0: T_{\text {finite }} ; \text { at } r \rightarrow \infty: T_{w}=0 \\
\text { at } t=0: T=T_{w}=0
\end{array}\right.
$$

To solve the system of equations (1) to (3) for a steady periodic regime, one have used Fourier's transformation introducing complex number $j: T=\widetilde{T} e^{2 \omega j t}$ for the temperature and $P=\widetilde{P} e^{2 \omega j t}$ for the volumic power. Therefore the partial differential equations (1) and (2) are transformed into second order differential equations with a source term that can be solved easily. The corresponding solution for the average temperature within the carbon fiber is finally:

where:

$$
\overline{\tilde{T}}=\frac{\tilde{P} R^{2}}{\alpha_{1}^{2} k_{r}}\left[1-\frac{2}{\varepsilon \alpha_{1} R}\right]
$$

$$
\begin{gathered}
\alpha_{1}=\frac{1+j}{\delta_{1}}, \quad \alpha_{2}=\frac{1+j}{\delta_{2}}, \quad \delta_{1}=\sqrt{\frac{k_{r}}{\omega \rho c}}, \quad \delta_{2}=\sqrt{\frac{k_{W}}{\omega \rho_{w} c_{w}}} \\
\text { and } \varepsilon=\frac{k_{r} \alpha_{1} K_{0}\left(\alpha_{2} R\right)}{k_{w} \alpha_{2} K_{1}\left(\alpha_{1} R\right)}+\frac{I_{0}\left(\alpha_{1} R\right)}{I_{1}\left(\alpha_{1} R\right)}
\end{gathered}
$$

In Eq.5, the quantities $I_{0}, I_{1}$ are modified Bessel functions of first kind and $K_{0}, K_{1}$ modified Bessel functions of second kind.

The relationship between the measured rms value of the voltage $V_{3 \omega}$ and the amplitude $\|\overline{\tilde{T}}\|$ of the average temperature of the fiber $\overline{\tilde{T}}$ (Eq.4) is given by:

$$
V_{3 \omega r m s}=I_{r m s} R_{0} \alpha_{e}\|\overline{\tilde{T}}\|
$$

where the product $R_{0} \alpha_{e}\|\overline{\tilde{T}}\|$ comes from the effect of the temperature on the electrical resistance $R_{X}$ of the carbon fiber $\left(R_{X}=R_{0}\left(1+\alpha_{e} T\right)\right)$. The temperature coefficient $\alpha_{e}=2.410^{-4} \mathrm{~K}^{-1}$ of the PAN/FT300B carbon fiber is more than 10 times smaller than the ones of metallic wires.

\subsection{D analytical model}

In fact as show on Fig.1, heat conduction might be 2D with not only radial but also with axial heat transfer within the carbon fiber. Therefore we have also developed a 2D axisymmetric thermal model as shown on Fig. 3 describing axial and also radial heat transfer within the whole fiber surrounded by de-ionized water. In order to take into account the anisotropy of the fiber one have considered two thermal conductivity components the radial one $k_{r}$ and also the axial one $k_{z}$. As previously a Joule heating with a volumic power $P$ is within the carbon fiber and the deionized water is considered as a semi-infinite medium. In addition for the $2 \mathrm{D}$ model, a temperature $(T=0)$ is prescribed on both sides at $z=0$ and $z=2 L$.

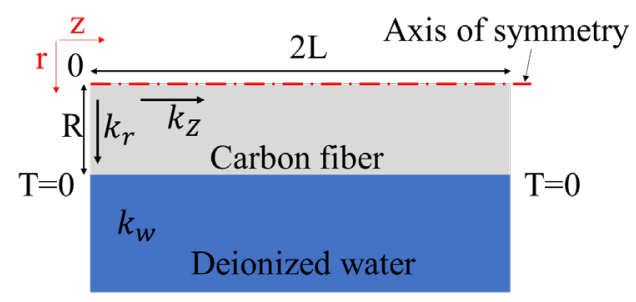

$\infty$

Fig. 3. Geometry for the $2 \mathrm{D}$ thermal model with a carbon fiber surrounded by de-ionized water

The governing heat conduction equations inside the fiber and the de-ionized water have the following form: 


$$
\begin{gathered}
k_{r}\left(\frac{\partial^{2} T}{\partial r^{2}}+\frac{1}{r} \frac{\partial T}{d r}\right)+k_{z} \frac{\partial^{2} T}{\partial z^{2}}-\rho c \frac{\partial T}{\partial t}=-P \\
k_{w}\left(\frac{\partial^{2} T_{w}}{\partial r^{2}}+\frac{1}{r} \frac{\partial T_{w}}{d r}\right)+k_{w} \frac{\partial^{2} T_{w}}{\partial z^{2}}-\rho_{w} c_{w} \frac{\partial T_{w}}{\partial t}=0
\end{gathered}
$$

where $T$ and $T_{W}$ are the temperatures inside the fiber and water respectively and $k_{z}$ is the axial thermal conductivity of the fiber.

The internal and external boundaries conditions are:

$$
\left\{\begin{array}{c}
\text { at } r=R: T=T_{w} \text { and }\left.k_{r} \frac{\partial T}{\partial r}\right|_{R}=\left.k_{w} \frac{\partial T_{w}}{\partial r}\right|_{R} \text { (perfect contact) } \\
\text { at } r=0: T_{\text {finite }} ; \text { at } r=\infty: T_{w}=0 \\
\text { at } z=0: T=T_{w}=0 ; \text { at } z=L: \frac{\partial T}{\partial z}=\frac{\partial T_{w}}{\partial z}=0
\end{array}\right.
$$

To solve this problem, one have used a double transformation of the temperature $T$ (and also for $T_{\mathrm{w}}$ ). First a complex Fourier transformation was used: $T=\tilde{T} e^{2 \omega j t}$ for the temperature and $P=\tilde{P} e^{2 \omega j t}$ for the volumic power. Secondly we applied the following finite sine transformation defined as [14]:

$$
\tilde{\theta}=\int_{0}^{L} \tilde{T} \sin \left(\beta_{n} z\right) d z
$$

The appropriate transformation is of sinus type because of the type of boundary condition (prescribed temperature) on both sides of z-axis [15].

With these two transformations, the temperature of the fiber in the transform space can be expressed as:

$$
\tilde{\theta}=a_{1} I_{0}\left(\gamma_{n} r\right)+\frac{\tilde{P}}{\beta_{n} k_{r} \gamma_{n}^{2}} \quad \text { with }\left\{\begin{array}{c}
\beta_{n}=\left(n+\frac{1}{2}\right) \frac{\pi}{L} \text { and } n=0,1,2 \ldots \infty \\
\gamma_{n}^{2}=\frac{k_{z}}{k_{r}}\left(\alpha_{z}^{2}+\beta_{n}^{2}\right) \text { with } \alpha_{z}^{2}=\frac{2 \rho c j \omega}{k_{z}}
\end{array}\right.
$$

where $\tilde{\theta}$ is the temperature transformed twice and $I_{0}$ represents a modified Bessel function. In Eq. $11, a_{1}$ is a term which will be computed further using boundary conditions.

Then after a first integration of $\tilde{\theta}$ over the radius $\mathrm{r}$ from 0 to $R$, one have used a quadrupole formulation [1] to take into account the bilayer shown in Fig. 3:

$$
\left[\begin{array}{l}
\overline{\tilde{\theta}} \\
\tilde{Q}
\end{array}\right]=\left[\begin{array}{ll}
A & B \\
C & D
\end{array}\right]\left[\begin{array}{c}
\tilde{\theta}_{R} \\
\widetilde{\Phi}_{R}
\end{array}\right]
$$

where $A, B, C, D$ and $\widetilde{Q}$ are defined as:

$$
\left\{\begin{array}{c}
A=1 \\
B=\frac{I_{0}\left(\gamma_{n} R\right)}{2 \pi k_{r} L \gamma_{n} R I_{1}\left(\gamma_{n} R\right)}-\frac{1}{\pi L k_{r} R^{2} \gamma_{n}^{2}} \\
C=\pi R^{2} L k_{r} \gamma_{n}^{2} \beta_{n} \\
D=\frac{I_{0}\left(\gamma_{n} R\right) \gamma_{n} \beta_{n} R}{2 I_{1}\left(\gamma_{n} R\right)} \\
\tilde{Q}=\pi R^{2} L \tilde{P}
\end{array}\right.
$$

In Eq. 12, $\widetilde{\theta}_{R}$ and $\widetilde{\Phi}_{R}$ are the temperature and heat flux located at $r=R$ in the transform space and are linked by the following relationship since water is considered as a semi-infinite medium [14]:

where:

$$
\widetilde{\theta}_{R}=Z_{\infty} \widetilde{\Phi}_{R} \text { with } Z_{\infty}=\frac{K_{0}\left(\gamma_{w n} R\right)}{2 \pi R L k_{w} \gamma_{w n} K_{1}\left(\gamma_{w n} R\right)}
$$




$$
\left\{\begin{array}{l}
\gamma_{w n}^{2}=\alpha_{w}^{2}+\beta_{n}^{2} \\
\alpha_{w}^{2}=\frac{2 \rho_{w} c_{w} j \omega}{k_{w}}
\end{array}\right.
$$

Finally, using the relationship for the inverse of the finite sine transformation which introduces the solution as a serie from $n=0$ to $\infty$ and by space integration along the axis of the fiber from 0 to $L$, one can obtain the complex value of the average temperature of the fiber:

$$
\overline{\overline{\tilde{T}}}=\left(\frac{2}{L^{2}} \sum_{n=0}^{\infty} \frac{\overline{\tilde{\theta}}}{\beta_{n}}\right) \text { with } \overline{\tilde{\theta}}=\left(\frac{A Z_{\infty}+B}{C Z_{\infty}+D}\right) \tilde{Q}
$$

where $I_{0}, I_{1}$ are modified Bessel functions of first kind and $K_{0}, K_{1}$ modified Bessel functions of second kind.

The relationship between the measured rms value of the voltage $V_{3 \omega}$ and the amplitude $\|\overline{\bar{T}}\|$ of the average temperature of the fiber $\overline{\overline{\tilde{T}}}$ (Eq. 16) is similar to the one given by Eq.6.

One should not that the 2D problem represented by Eq. 12 can be seen (see Fig.4) as an equivalent electrical network using the following two impedances defined by [14]:

$$
Z_{2}=\frac{D-\beta_{n}}{C} \text { and } Z_{3}=\frac{\beta_{n}}{C}
$$

where in the bottom line of Eq.12 the quantities $C, D$ and $\widetilde{Q}$ have been divided by $\beta_{n}$ since the determinant $A D$ $B C$ of the matrix is not equal to $1\left(A D-B C=\beta_{n}\right)$.

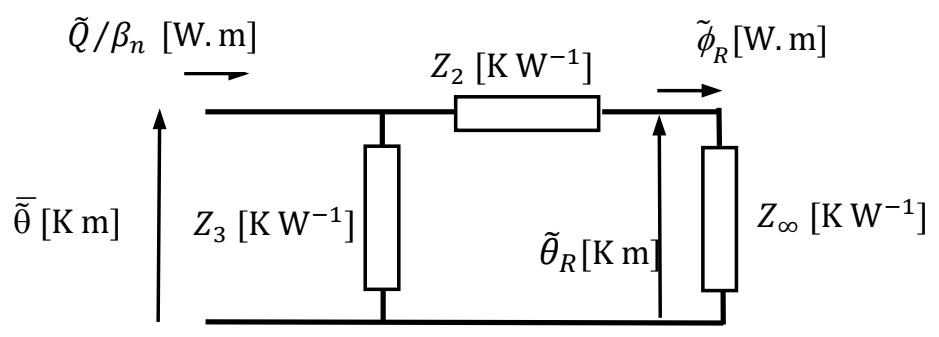

Fig. 4. Representation of the $2 \mathrm{D}$ thermal model using impedances

\section{Comparison between analytical 1D, 2D models and a 2D numerical model}

The values of voltage $V_{3 \omega}$ predicted with the two previous 1D and 2D thermal models are now compared with a 2D numerical model computed with finite element (COMSOL software). For this simulation, one have considered a modulated current of $1 \mathrm{~mA}$ amplitude crossing a carbon fiber of $1.7 \mathrm{~mm}$ length, $7 \mu \mathrm{m}$ diameter and of $900 \Omega$ electrical resistance with a $\alpha_{e}=2.410^{-4} \mathrm{~K}^{-1}$ temperature coefficient. The thermal properties are axial thermal conductivity of $10.47 \mathrm{Wm}^{-1} \mathrm{~K}^{-1}$ and volumic heat capacity of $3.910^{6} \mathrm{Jm}^{-3} \mathrm{~K}^{-1}$, both are coming from Mishra [6]. In addition a radial thermal conductivity of $0.8 \mathrm{Wm}^{-1} \mathrm{~K}^{-1}$ was chosen. 


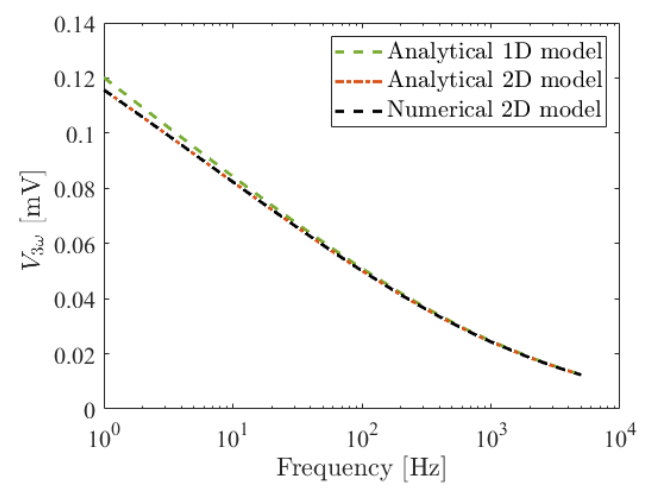

Fig 5. Voltage $V_{3 \omega}$ versus frequency for the analytical $1 \mathrm{D}$ and 2D models and comparison with a 2D numerical model computed with finite elements.

Results in Fig. 5 shows a good agreement between the 2D analytical and 2D numerical models (less than 0.1\% discrepancy). One can notice that the 1D analytical thermal model does not fit the 2D ones especially at low frequency between 1 and $100 \mathrm{~Hz}$, the discrepancy is about $8 \%$ at $1 \mathrm{~Hz}$. As the 1D model was used in a previous work [5] one has tried to estimate the bias when estimating the radial thermal conductivity using only the 1D thermal model. For this purpose one have simulated data $\left(V_{3 \omega}\right.$ vs $\left.f\right)$ using the $2 \mathrm{D}$ analytical model and a value of radial thermal conductivity was estimated using the $1 \mathrm{D}$ analytical model. The estimation of $k_{r}$ was performed under MATLAB software using simplex method. The results are presented in Fig.6 for $k_{r}=1 \mathrm{Wm}^{-1} \mathrm{~K}^{-1}$ and $k_{z}=10 \mathrm{Wm}^{-}$ ${ }^{1} \mathrm{~K}^{-1}$ and in Table 2 with various sets of $k_{r}$ and $k_{z}$ values.

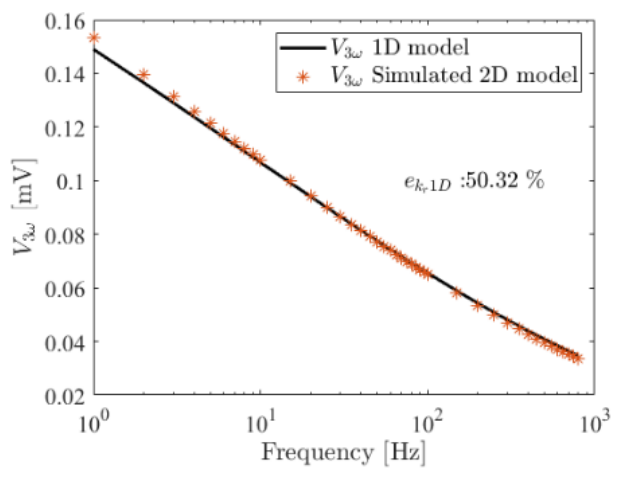

Fig. 6. Computation of the bias $e_{k r I D}$ on the estimated radial conductivity by using the $1 \mathrm{D}$ analytical model (simulated data using the 2D analytical model with $k_{z}=10 \mathrm{Wm}^{-1} \mathrm{~K}^{-1}$ and $k_{r}=1 \mathrm{Wm}^{-1} \mathrm{~K}^{-1}$ )

Table 2. Bias on the estimation of $k_{r}$ using the 1D analytical model, data are simulated using the 2D analytical model and various set of $k_{r}$ and $k_{z}$ values

\begin{tabular}{cccc}
\hline $\begin{array}{c}k_{r} \\
\mathrm{Wm}^{-1} \mathrm{~K}^{-1}\end{array}$ & $\begin{array}{c}k_{z} \\
\mathrm{Wm}^{-1} \mathrm{~K}^{-1}\end{array}$ & $k_{z} / k_{r}$ & $\begin{array}{c}e_{k r I D} \\
\%\end{array}$ \\
\hline 0.1 & 5 & 50 & 0.48 \\
0.2 & 10 & 50 & 1.93 \\
0.5 & 5 & 10 & 8.03 \\
1.0 & 10 & 10 & 50.32 \\
\hline
\end{tabular}

Fig. 5 shows the agreement between calculated $V_{3 \omega}$ value using 1D analytical model for the estimation of $k_{r}$ value and simulated data $V_{3 \omega}$ using the $2 \mathrm{D}$ analytical model. The agreement between $V_{3 \omega}$ values after the estimation process is correct and the bias $\mathrm{e}_{\mathrm{krID}}$ is very large (50.32\%). Table 2 shows more values of the bias $\mathrm{e}_{\mathrm{kr} 1 \mathrm{D}}$ for various sets of $k_{r}$ and $k_{z}$ values. For small values of $k_{r}$, the bias $\mathrm{e}_{\mathrm{kr1D}}$ is quite small which is probably due to high value of the radial thermal gradient compared to the axial one. For higher values of $k_{r}$ (which will be the case in our experimental results shown in section 7) the bias $\mathrm{e}_{\mathrm{krID}}$ is large. This suggest that despite the use of water around 
the fiber in order to increase radial heat transfer, there exists still some axial heat transfer responsible for the observed bias $e_{k r I D}$ obtained using only a $1 \mathrm{D}$ model. As a conclusion of this section, in order to estimate $k_{r}$ value, it is much more appropriate to use the 2D model (Eq.16) than the 1D one.

\section{Sensitivity analysis}

The measurement of the radial thermal conductivity is not easy and it is important to perform a sensitivity analysis which is very useful to choose the best operating conditions in order to perform parameter estimation with the lowest uncertainty. Sensitivity coefficients of the measured temperature $T$ with respect to the parameter $u_{p}$ (in our case $k_{r}$ or $k_{z}$ ) are defined by:

$$
X_{u_{p}}=u_{p} \frac{\partial T}{\partial u_{p}} \text { or } X_{u_{p}}^{*}=\frac{u_{p}}{T_{\max }} \frac{\partial T}{\partial u_{p}} \text { with } u_{p}=k_{r}, k_{z}
$$

The derivative $\partial \mathrm{T} / \partial \mathrm{u}_{\mathrm{p}}$ was computed using the $2 \mathrm{D}$ analytical model described previously and the geometry and thermal properties values considered at the beginning of previous section.

Fig. 7 is very interesting since it compares the sensitivities to $k_{r}$ and to $k_{z}$. In addition, we have added the value of the sensitivity to $k_{z}$ during axial thermal conductivity of fiber (under vaccum) as done by Mishra [6], the latter is indicated with the notation "with $k_{w}=0$ " corresponding to an adiabatic condition. One can show that the use of deionized water around the carbon fiber greatly reduces the sensitivity to $k_{z}$ almost to 0 and increases the one to $k_{r}$ which was expected. However, the sensitivity to $k_{r}$ still remains modest $\left(X_{k r}=0.29^{\circ} \mathrm{C}\right)$ which will induce larger uncertainty on $k_{r}$ compared to the one for $k_{z}$ estimation under vacuum [6]. One can also notice that the sensitivity to $k_{r}$ does not depend much on the frequency within the 1 to $1000 \mathrm{~Hz}$ range.

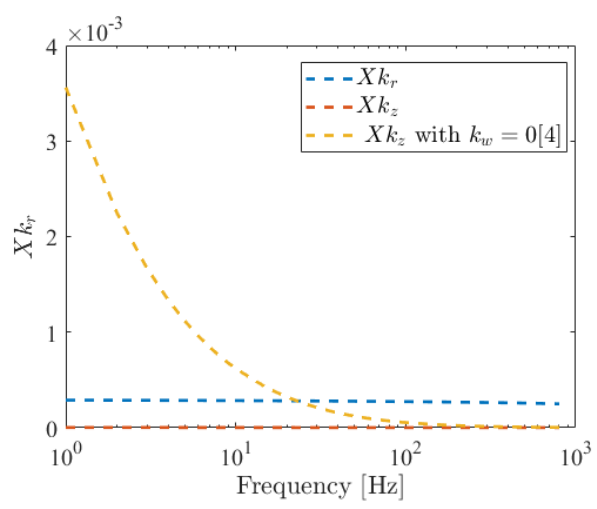

Fig. 7. Sensitivity analysis of the $3 \omega$ voltage to the radial and axial thermal conductivities

A question which arises is whether we can further increase the sensitivity to $k_{r}$ by replacing water with another fluid or material around the carbon fiber? Fig. 8 shows the effect of thermal conductivity of the surrounding material on the sensitivity coefficient of the fiber temperature with respect to $k_{r}$. There is clearly a gain with a 6 times increase of the sensitivity coefficient to $k_{r}$ for a 10 times increase of the thermal conductivity $k_{w}$ of the surrounding material (from 0.59 to $5.9 \mathrm{~W} \cdot \mathrm{m}^{-1} \cdot \mathrm{K}^{-1}$ ). However, practically it is difficult to find a fluid or a material with such properties while ensuring good thermal contact with the carbon fiber. By cooling the water one could obtain ice with a much higher thermal conductivity $\left(2.1 \mathrm{Wm}^{-1} \mathrm{~K}^{-1}\right.$ at $\left.0^{\circ} \mathrm{C}\right)$ than the one of water but the specific heat capacity would drop (from $4.22 \mathrm{kJkg}^{-1} \mathrm{~K}^{-1}$ at room temperature to $2.06 \mathrm{~kJ} \mathrm{~kg}^{-1} \mathrm{~K}^{-1}$ at $0{ }^{\circ} \mathrm{C}$ ) which would not bring much gain in terms of sensitivity to $k_{r}$. Indeed the relevant quantity for choosing a surrounding medium providing a heat sink effect is its thermal effusivity which is the square root of the $k_{w} \rho_{w} c_{w}$ product. Therefore we haven't found yet better than de-ionized water. 


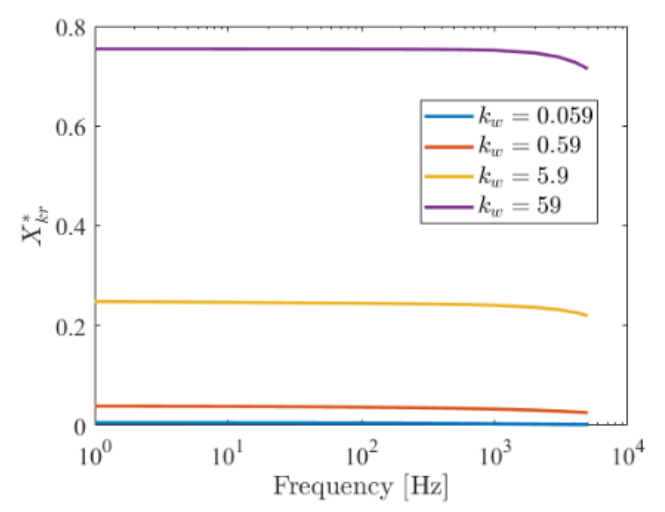

Fig. 8. Effect of the thermal conductivity $k_{w}$ of the surrounding material on the sensitivity coefficient of the fiber temperature with respect to its radial thermal conductivity $k_{r}$

\section{Experimental setup, samples and uncertainty analysis}

\subsection{Experimental setup}

In the $3 \omega$ method, the average temperature of the sensor or of the sample itself is measured thanks to the variation of its electrical resistance versus temperature. However this variation is small and a specific electronic device is required to isolate this contribution before enhancing the signal using a lock-in amplifier (LIA). For this purpose, the electronic device can be a Wheatstone bridge or a circuit with differential amplifiers. The Wheatstone bridge works fine for sensor or sample of low electrical resistance (less than $400 \Omega$ ) not so far from the input impedance of LIA [6]. For higher electrical resistance the use of differential amplifiers is required which corresponds to our case, the electrical resistance of PAN type carbon fiber of $1.5 \mathrm{~mm}$ length being about $900 \Omega$. In our device as shown in Fig. 9, two differential amplifiers (AD624) are used to get the difference of voltage between the resistance $R_{X}$ of the sample and the one $R_{V}$ of a reference resistance (adjustable until $5 \mathrm{k} \Omega$ ). The adjustment of the latter is performed with a $1 \omega$ pulsation using the LIA (Ametek 7265) and then $V 3 \omega$ measurement which contains the temperature information is performed with a $3 \omega$ pulsation.

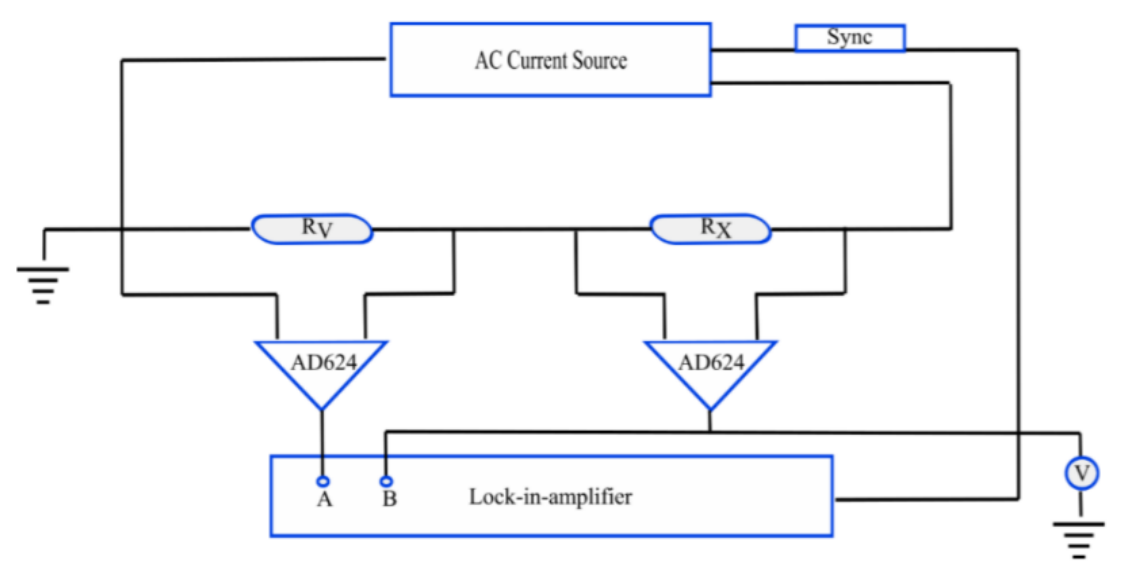

Fig. 9. Schematic electrical setup for $3 \omega$ voltage measurement. 
The sample holder is built using two small sections of an FR4 electronic circuit board covered with $15 \mu \mathrm{m}$ of copper which are glued on a polycarbonate block (Fig. 10). The ends of the carbon fiber are held on the copper parts with silver paste insuring the required prescribed temperature boundary condition at $z=0$ and $z=2 \mathrm{~L}$ (see Eq. 9), then the whole is immersed in deionized water.

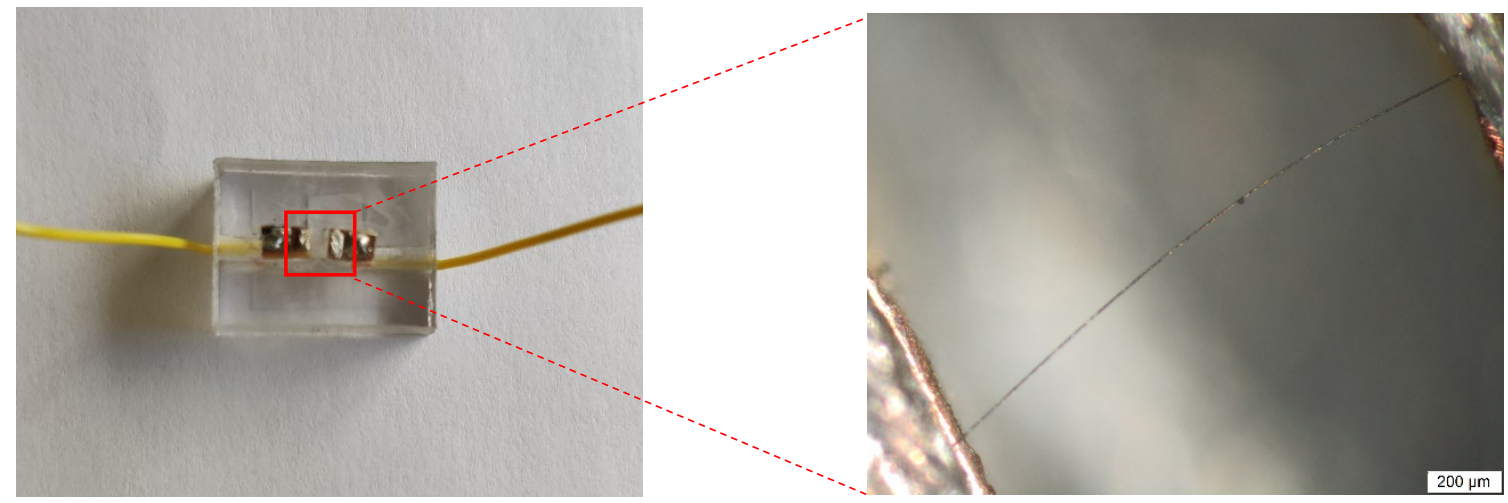

Fig. 10. Sample holder and zoom on the carbon fiber implementation

The electrical resistance of the fiber is measured before and after been submerged to ensure there is no electrical leakage due to any contamination of the deionized water. Table. 4 presents the geometric and electrical characteristics of the three carbon fibers used for performing the experiments. These values are presented as a range in order to perform the uncertainty analysis on $k_{r}$ estimation (see next section).

Table 4. Characteristics of the PAN type carbon fibers FT300B used for $k_{r}$ measurements

\begin{tabular}{cccc}
\hline Carbon fiber & Diameter $/ \mu \mathrm{m}$ & Length $/ \mathrm{mm}$ & Electr. resistance $R_{0} / \Omega$ \\
\hline Sample 1 & $6.39-8.52$ & $1.68-1.71$ & 1043.2 \\
Sample 2 & $7.66-9.51$ & $1.73-1.74$ & 867.6 \\
Sample 3 & $6.33-8.59$ & 0.74 & 494.8 \\
\hline
\end{tabular}

The $V_{3 \omega}$ voltage measurements are carried out over a wide frequency range ( 1 to $800 \mathrm{~Hz}$ ) with approximately 42 frequency values with moreover a few higher frequencies (around $5 \mathrm{kHz}$ ) to obtain the asymptotic values.

\subsection{Uncertainty analysis}

There are many sources of uncertainty when measuring $k_{r}$. One can cite the quantities such as length, diameter of fibers, electric current and voltage as well as the thermal parameters assumed to be known $k_{z}, \rho c \ldots$ The principle of calculating the overall uncertainty on the estimated radial thermal conductivity $k_{r}$ is issued from previous reference books dealing with parameter estimation [16, 17]. The absolute uncertainties $1.96 \sigma_{k r}$ on the estimated $k_{r}$ with $95 \%$ confidence bands comes from the computation of the matrix of variance-covariance $S_{\text {final }}$ at the final iteration as proposed by Milosevic [18] and defined by:

$$
S_{\text {final }}=\left[X^{T} W X\right]^{-1} \text { with } W=\left[\sigma_{V 3 \omega}^{2}+\sum_{P}\left(\sigma_{m_{P}} \frac{\partial V_{3 \omega}}{\partial m_{P}}\right)^{2}\right]^{-1}
$$

where $X$ is the sensitivity coefficient matrix, $W$ is the variance covariance matrix, $\sigma_{V 3 \omega}$ is the standard deviation of the measured voltage $V_{3 \omega}, \sigma_{m_{P}}$ is the standard deviation of the known parameters $m_{p}$. The values of the standard deviation $\sigma_{m p}$ are found from the relative error $e_{m p}$ on the known parameters listed in Table $5\left(e_{m p}=1.96 \sigma_{m p} / m_{p}\right)$.

Table 5. Known parameters and their relative uncertainties used for uncertainty calculation over $k_{r}$

\begin{tabular}{cc}
\hline Parameters $m_{p}$ & Relative error $e_{m p} / \%$ \\
\hline Current $I_{0}$ & 0.1 \\
Electrical resistivity $R_{0}$ & 0.2 \\
Temp. coeff. of resistance $\alpha_{e}$ & 3 \\
Length of the carbon fiber $L$ & 9 \\
Radius of the carbon fiber $R$ & 13 \\
Axial thermal conductivity of the carbon fiber $k_{z}$ & 8.1 \\
Volumic heat capacity of the carbon fiber $\rho c$ & 4.9 \\
\hline
\end{tabular}


Finally, the typically value of the error for the estimated value of radial thermal conductivity is $24 \%$. This error is higher than the one obtained for the axial thermal conductivity (about 8.1\% [6]) because heat transfer is studied along a much shorter distance which is measured with lower accuracy.

\section{Experimental results}

The estimation of the radial thermal conductivity was carried out using the $2 \mathrm{D}$ analytical thermal model validated previously. The estimation procedure has consisted in minimizing the sum of square of the difference between computed and measured voltage $V_{3 \omega}$ and this using simplex method. The value of $k_{z}$ and $\rho c$ for the studied fiber were considered known (coming from a previous work [6]).

Fig. 11a shows a good agreement between the measured and calculated values of the voltage $V_{3 \omega}$ after the estimation of the radial thermal conductivity $k_{r}$. To improve the estimation, we have in fact considered from an experimental point of view the differences between measured voltages and their asymptotic value obtained at high frequencies around $5 \mathrm{kHz}$, the asymptotic value should in theory be zero. Indeed, it was found that from one test to another this asymptotic value was not always equal to zero, indicating the presence of a slight electronic "offset".

Fig. $11 \mathrm{~b}$ shows the residuals between measured and computed $V_{3 w}$ values after the estimation of $k_{r}$. It appears that the maximum value of the residuals is about $0.06 \mathrm{mV}$ for a maximum voltage of $2.3 \mathrm{mV}$ which in relative magnitude gives residuals smaller than $2.6 \%$ which remains low.

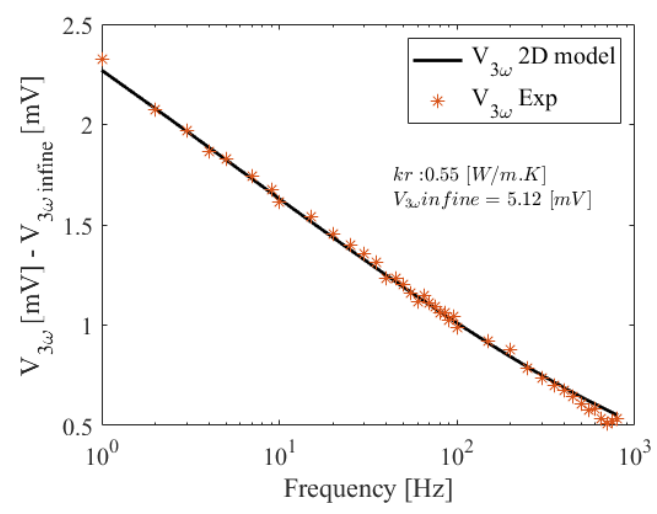

(a)

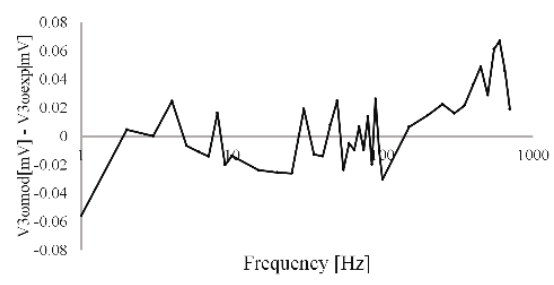

(b)

Fig 11. (a) Measured and computed $V_{3 \omega}$ voltages after the estimation of the radial thermal conductivity (sample\#1, FT300B carbon fiber) (b) $V 3 \omega$ residuals

Table 6 shows the results for three different PAN / FT300B type carbon fibers with similar geometric characteristics. The estimated values of the radial thermal conductivity ranges from 0.4 to $0.8 \mathrm{Wm}^{-1} \mathrm{~K}^{-1}$, they are more than 10 times lower than the axial value equal to $10.5 \mathrm{Wm}^{-1} \mathrm{~K}^{-1}$ of the same batch of fiber [6], this shows the highly anisotropic structure of such PAN type carbon fibers. These results are in agreement with discussion in paper from Hind [19] where a thermal conductivity ratio from 5 to 10 is mentioned for PAN type carbon fibers. 
Table 6. Measured radial thermal conductivity $k_{r}$ for a PAN type carbon fiber FT300B

\begin{tabular}{cccc}
\hline $\begin{array}{c}\text { Radial thermal } \\
\text { conductivity }\end{array}$ & Sample 1 & Sample 2 & Sample 3 \\
\hline$k_{r}, \mathrm{Wm}^{-1} \mathrm{~K}^{-1}$ & 0.549 & $0.435 / 0.652^{*}$ & 0.801 \\
\hline
\end{tabular}

* Two measurements have been performed on the same carbon fiber

\section{Effect of thermal contact resistance and of the convection in water}

The numerical and analytical models used above works under two strong hypotheses: 1. perfect contact between carbon fiber and electrode copper, 2. negligible convection losses. The first hypothesis is justified by the fact that the heat exchanges would occur mainly in the radial direction of the fiber thanks to the presence of deionized water, then the heat flow towards the extremities of the fiber decreases strongly. While the second hypothesis is supported by the argument that the heat generated is not constant, it oscillates according to the frequency at which the system is exposed, these oscillations do not allow the medium to heat up constantly, so that its temperature rises are drastically reduced, thus preventing convection in the water. In order to justify those hypothesis, a numerical study is dedicated to the verification of those hypothesis.

The value of the thermal contact resistance between the copper block / carbon fiber connection (fiber type: PAN FT300B) was measured in a previous investigation giving a value of $8.8310^{-6} \mathrm{~m}^{2} \mathrm{~K} \mathrm{~W}^{-1}$ (Mishra et al. [6]). This value was taken into account in a numerical model with the aim of estimating the influence of this thermal resistance on the final value of the radial thermal conductivity. The induced bias on the radial thermal conductivity was finally about of $6.4 \%$ with is less that the relative uncertainty on $k_{r}$ measurement.

Then, two studies have been carried out with the aim of estimating the influence of convection under steady state conditions and then subject to harmonic heating.

For this purpose, we have performed finite element calculations using Comsol software. Two different types of simulations were carried out in each study, the first under the influence of (only) conduction in water and the second subject to the convective phenomenon in water. The conduction was performed using the "Heat transfer in solids" module of Comsol and the convection using the "Conjugate heat transfer fluid-solid" module which allows the interaction between the energy equation and the Navier-Stokes equations. The harmonic and convective study was performed using transient regime and very small time steps, each time period being discretized with 10 time steps.

As a first step, the steady state study showed a difference in the mean temperature of the carbon fiber. With convection inside the water, this mean temperature is about $1.46 \%$ lower than the one obtained with only conduction (see Table 7 below). As in section 4, a bias on the $V_{3 \omega}$ voltage with a few percentage might induce a substantial bias in the estimated radial thermal conductivity. Thus we have performed the harmonic study which is closer to our experimental conditions for $k_{r}$ measurement. As shown in Table 7 below, the discrepancy between the average temperature of the fiber between conduction and convection in water is much smaller typically less than $0.23 \%$ for a frequency higher than $1 \mathrm{~Hz}$. At this level the induced bias on the $k_{r}$ measurement is small. Indeed, by changing the average temperature of the carbon fiber about $0.23 \%$ we have obtained a $k_{r}$ variation about $4 \%$ which is smaller than the computed relative uncertainty of $k_{r}$ measurement (24\% see section 6.2 ). In addition this $0.23 \%$ change of temperature which would involve a $0.23 \%$ change of the $V_{3 \omega}$ voltage is not detectable with the lock in amplifier as for voltage about $1 \mathrm{mV}$ the accuracy is less than $0.3 \%$ as mentioned in the datasheet of our lock-in Amplifier [20]).

Table 7 Average temperature of the carbon fiber with a) only conduction and b) with convection in water.

\begin{tabular}{cccc}
\hline $\mathrm{f}$ & $\bar{T}_{\text {Convection }}$ & $\begin{array}{c}\bar{T}_{\text {Conduction }} \\
\mathrm{K}\end{array}$ & $e^{\%}$ \\
\hline Steady state & 2.17023 & 2.20244 & 1.46 \\
1 & 3.73542 & 3.74401 & 0.23 \\
5 & 2.99306 & 2.99927 & 0.21 \\
10 & 2.66869 & 2.67273 & 0.15 \\
\hline
\end{tabular}

with $e=100 * \frac{\bar{T}_{\text {Convection }}-\bar{T}_{\text {Conduction }}}{T_{\text {Condution }}}$ 
Also in Table 7 we can see that the convective effect leads always to smaller temperatures as expected. In addition, as the frequency increases, the bias $e$ on average temperatures decays bringing the mean temperature values of the fiber subject to conduction and convection closer to each other.

One have also computed the value of Rayleigh number for the steady state case. The Rayleigh number is less than 11 which is much lower that the threshold value of 1100 under which we can neglect convective effect [21]

\section{Conclusions}

An analytical 2D thermal model in steady periodic regime has been developed and validated in order to estimate the radial thermal conductivity of carbon fiber. It appeared that the use of a 1D thermal model as mentioned in the work of Liang's work [5] could lead to bias on $k_{r}$ value of the order of $50 \%$. In addition, the presence of water around the carbon fiber has been shown to induce much higher sensitivity of the measured $V_{3 \omega}$ voltage with respect to the radial conductivity $k_{r}$ while decreasing the one with respect to the axial conductivity $k_{z}$.

Three different samples of PAN type carbon fibers (FT300B) with a diameter between 7.4 and $8.6 \mu \mathrm{m}$ were characterized and the estimated radial thermal conductivity values were found between 0.43 and $0.80 \mathrm{Wm}^{-1} \mathrm{~K}^{-1}$.

The induced bias on the radial thermal conductivity produced by the thermal contact resistance between the copper block / carbon fiber connection was finally about of $6.4 \%$ with is less that the relative uncertainty on $k_{r}$ measurement.

Under steady state conditions, convection could generate some variations in the mean temperature of the carbon fiber of around $1.4 \%$. however, a have performed harmonic study which is closer to our experimental conditions for $k_{r}$ measurement shows a discrepancy between the average temperature of the fiber between conduction and convection in water is much smaller typically less than $0.23 \%$ which would involve a $0.23 \%$ change of the $V_{3 \omega}$ voltage for a frequency higher than $1 \mathrm{~Hz}$, a value thath is not detectable with the lock in amplifier.

Knowing the axial and radial of thermal conductivities of anisotropic carbon fibers of PAN type, future work will concern the measurement of the thermal contact resistance (TCR) between two carbon fibers. This would allow to provide all small scale information in order to predict or calculate the effective conductivity of polymer matrix composites reinforced with carbon fiber, but the measurement of fiber / fiber TCR is another challenge.

\section{References}

[1] J. Wang, X. Zhang, G.-P. Wu, Simultaneous measurements of thermal properties of individual carbon fibers, Int. J. Thermophys. 32 (2011) 974-983. https://doi.org/10.1007/s10765-011-0961-7

[2] H. S. Huang, S. Ganguli, A. K. Roy, Prediction of the transverse thermal conductivity of pitch-based carbon fibers, J. of Comp. Mater. 48, 111 (2014) 1383-1390. https://doi.org/10.1177/0021998313486501

[3] R. Wang, H. Zobeiri, H. Lin, W. Qu, X. Bai, Anisotropic thermal conductivities and structure in lignin-based microscale carbon fibers, Carbon 147 (2019) 58-69. https://doi.org/10.1016/j.carbon.2019.02.064

[4] J. L. Wang, M. Gu, X. Zhang.Y. Song, Thermal conductivity measurement of an individual fiber using a T type probe method, J. Phys. D-Appl. Phys. 42,110 (2009) 105502. http://dx.doi.org/10.1088/0022$\underline{3727 / 42 / 10 / 105502}$

[5] J. Liang, Experimental measurement and modeling of thermal conductivities of carbon fibers and their composites modified with carbon nanofibers, phD, University of Oklahoma, 2014.

[6] K. Mishra, B. Garnier, S. Le Corre, N. Boyard, Accurate measurement of the longitudinal themal conductivity and volumetric heat capacity of single carbon fibers with the $3 \mathrm{w}$ method, Therm. Analysis Calorim. 137, 14 (2019) 1037-1047. https://doi.org/10.1007/s10973-019-08568-Z

[7] B. Bouyer, X. Tardif, C. Mercader, D. Delaunay, Design of a new device for fibers strand axial thermal conductivity measurement, Int. J Therm. Sci. 161 (2020) 106740.

https://doi.org/10.1016/j.ijthermalsci.2020.106740 
[8] C. Pradere, J. C. Batsale, J. M. Goyhénèche, R. Pailler, S. Dilhaire, Thermal properties of carbon fibers at very high temperature, Carbon 47 (2009) 737-743. https://doi.org/10.1016/j.carbon.2008.11.015

[9] D. G. Cahill, Thermal conductivity measurement from 30 to $750 \mathrm{~K}$ : the 3omega method,» Review of Scientific Instruments 61 (1990) 802-808. https://doi.org/10.1063/1.1141498

[10] L. Lu, W. Yi, D. L. Zhang, 3 omega method for specific heat and thermal conductivity measurements, Rev. Sci. Instrum., 72 (2001) 2996-3003. https://doi.org/10.1063/1.1378340

[11] C. Xing, C. Jensen, T. Munro, B. White, H. Ban, M. Chirtoc, Thermal property characterization of fine fibers by the 3-omega technique, Appl. Thermal Eng., 71, 1 (2014) 589-595.

https://doi.org/10.1016/j.applthermaleng.2014.06.022

[12] T. Ding, Y. Jannot, V. Schick, A. Degiovanni, Analysis of the non-linearity of the heat transfer equation in case of a time-dependent heat source: application to the 3 omega method, J. Eng. Mathematics 121 (2020) 85-99. http://dx.doi.org/10.1016/j.ijthermalsci.2014.06.010

[13] J. Liu, W. Qu, Y. Xie, B. Zhu, T. Wang, X. Bai, X. Wang, Thermal conductivity and annealing effect on structure of lignin-based microscale carbon fibers, Carbon 121(2017) 35-47. https://doi.org/10.1016/j.carbon.2017.05.066

[14] D. Maillet, S. André, J. C. Batsale, A. Degiovanni, C. Moyne, Thermal quadrupoles - Solving the heat equation through integral transforms, Wiley, Chichester, 2000.

[15] H. S. Carslaw, J. C. Jeager, Conduction of heat in solids, $2^{\text {nd }}$ ed., Oxford Univ. Press, Bristol, 1959.

[16] J.V. Beck, K.J. Arnold, Parameter Estimation in Engineering and Science, Wiley 1977

[17] B. Remy, S. Andre, Chap. 9 -Nonlinear estimation techniques, Thermal measurements and inverse techniques, ed. H.R.B. Orlande, O. Fudym, D. Maillet, R. M. Cotta, CRC Press, Serie Heat Transfer 2011.

[18] N. D. Milosevic , M. Raynaud, K. D. Maglic, Estimation of thermal contact resistance between the materials of double-layer sample using the laser flash method, Inv. Prob. in Eng., 10 (2002) 85-103. https://doi.org/10.1080/10682760290022528

[19] S. Hind, F. Robitaille, Measurement, modeling and variability of thermal conductivity for structural polymer composites, Polym. Comp., 31 (2010) 847-857. https://doi.org/10.1002/pc.20867

[20] Ametek, "Signal recovery - Model 7265 DSP Lock-in Amplifier", 2013. [Online].

[21] O. G. Martynenko et P. P. Khramtsov, Free Convective Heat Transfer - With many photographs of flows and heat exchage, Springer, 2005. 